\title{
Analysis of synchronization on time scale
}

\author{
Qingshui Liao ${ }^{\mathrm{a}, *}$ \\ Guizhou Normal University, Guiyang, Guizhou, China \\ a Email:qsliao_math@163.com \\ *Corresponding author
}

Keywords: Time scale, synchronization, Lyapunov stability theory.

\begin{abstract}
In this paper, we have studied the synchronization on time scale and given two theorems to illustrate the phenomenon. Furthermore, corresponding examples are presented based on the results. Numerical simulations show their feasibility. From a theoretical perspective, such a synchronization strengthen the security of communication, which could be considered as a good method for designing a more secure communication scheme in practical engineering applications.
\end{abstract}

\section{Introduction}

Recently, the phenomenon of synchronization in stochastic systems has attracted much interest (see for example [1-5]). Meanwhile, chaos synchronization is deemed to have a great potential in a large amount of application, arranging from secure communication to brain activity, even to optimization of nonlinear system performance.

As well known, both continuous and discrete systems are also important in implementing and applications. But it is troublesome to study the stability for continuous and discrete systems, respectively. Therefore, it is meaningful to study that on time scale which can unify the continuous and discrete situations [6-16]. To the best of our knowledge, the synchronization on time scale has not been studied. In this paper, we study it by using the time scale calculus theory and Lyapunov stability theory.

The rest of the paper is organized as follows: In section 2, some basic notions and some results are collected; In section 3, the synchronization on time scale is discussed.

\section{Preliminaries}

In this section, we briefly recall some basic definitions and results concerning time scale. Further general details can be found in [17].

Definition 2.1 [6] A time scale is arbitrary nonempty closed subset of the real set $\mathrm{R}$ with the topology and ordering inherited from $\mathrm{R}$.

Definition 2.2 [6] Let $t \in \mathrm{R}$, we define the forward jump operator $\sigma: \mathrm{T} \rightarrow \mathrm{T}$ by

$$
\sigma(t):=\inf \{s \in \mathrm{T}: s>t\} \text { for all } t \in \mathrm{R},
$$

while the backward jump operator $\rho: \mathrm{T} \rightarrow \mathrm{T}$ by

$$
\rho(t):=\sup \{s \in \mathrm{T}: s<t\} \text { for all } t \in \mathrm{R} \text {. }
$$

In this definition, we put $\inf \varnothing=\sup \mathrm{T}$ (i.e., $\sigma(M)=M$ if $\mathrm{T}$ has a maximum $M$ ) and $\sup \varnothing=\inf \mathrm{T}$ (i.e., $\rho(m)=m$ if $\mathrm{T}$ has a minimum $m$ ), where $\varnothing$ denotes the empty set. If $\sigma(t)>t$, we say that $t$ is right-scatted, while $\rho(t)<t$ we say that $t$ is left-scatted. Also, if 
$t<\sup \mathrm{T}$ and $\sigma(t)=t$, then $t$ is called right-dense, while if $t>\inf \mathrm{T}$ and $\sigma(t)=t$, then $t$ is called left-dense. Points that are right-scattered and left-scattered at the same time are called isolated. Points that are right-dense and left-dense at the same time are called dense. If $\mathrm{T}$ has a left-scattered maximum $M$, then we define $\mathrm{T}^{K}=\mathrm{T}-M$, otherwise $\mathrm{T}^{K}=\mathrm{T}$. The graininess function $\mu: \mathrm{T} \rightarrow[0, \infty)$ is defined by

$$
\mu(t):=\sigma(t)-t \text { for all } t \in \mathrm{R} .
$$

Definition 2.3 [6] Assume $f: \mathrm{T} \rightarrow R$ is a function and let $t \in \mathrm{T}^{K}$. Then we define $f^{\Delta}(t)$ to be the number (provided if exists) with the property that given any $\varepsilon>0$, there is a neighbourhood $U$ of $t$ (i.e., $U=(t-\delta, t+\delta) \bigcap \mathrm{T}$ for some $\delta>0)$ such that

$$
\left|f(\sigma(t))-f(s)-f^{\Delta}(\sigma(t)-s)\right| \leq|\sigma(t)-s| \text {,for all } s \in U
$$

We call the $f^{\Delta}(t)$ to be the delta (or Hilger) derivative of $f$ at $t$.

Lemma 2.1 [6] Assume $f: \mathrm{T} \rightarrow R$ is function and let $t \in \mathrm{T}^{K}$. We have the following

(i) If $f$ is differentiable at $t$, then $f$ is continuous at $t$.

(ii) If $f$ is continuous at $t$ and $t$ is right-scattered, then $f$ is continuous at $t$ with

$$
f^{\Delta}(t)=\frac{f(\sigma(t))-f(s)}{\mu(t)} .
$$

(iii) If $t$ is right-dense, then $f$ is differentiable at $t$ if $\lim _{s \rightarrow t} \frac{f(t)-f(s)}{t-s}$ exists as a finite number. In this case,

$$
f^{\Delta}(t)=\lim _{s \rightarrow t} \frac{f(t)-f(s)}{t-s} .
$$

Lemma 2.2 [6] Assume $f, g: \mathrm{T} \rightarrow R$ is differentiable at $t \in \mathrm{T}^{K}$. Then

(i) For any constant $a$ and $b$, the sum $a f+b g: \mathrm{T} \rightarrow R$ is differentiable at $t$ with

$$
(a f+b g)^{\Delta}(t)=a f^{\Delta}(t)+b g^{\Delta}(t) \text {. }
$$

(ii) If $f^{\Delta}(t)$ exists, then

$$
f(\sigma(t))=f(t)+\mu(t) f^{\Delta}(t)
$$

(iii) The product $f g: \mathrm{T} \rightarrow R$ is differentiable at with

$$
(f g)^{\Delta}(t)=f^{\Delta}(t) g(t)+f(\sigma(t)) g^{\Delta}(t)=f(t) g^{\Delta}(t)+f^{\Delta}(t) g(\sigma(t)) .
$$

Definition 2.4 [6] Assume $f: \mathrm{T} \rightarrow R$ is called rd-continuous provided it is continuous at rightdense points in $\mathrm{T}$ and its left-sided limits exist (finite) at left dense points in $\mathrm{T}$. The set of rdcontinuous functions $f: \mathrm{T} \rightarrow R$ is denoted by

$$
\mathrm{C}_{\mathrm{rd}}=\mathrm{C}_{\mathrm{rd}}(\mathrm{T})=\mathrm{C}_{\mathrm{rd}}(\mathrm{T}, R) \text {. }
$$

\section{The analysis of synchronization on time scale}

In this section, we consider a nonlinear dynamic systems arbitrary time scale T. Described by

$$
\left\{\begin{array}{c}
x^{\Delta}=a(t) x(\sigma(t))+b(t) y(t), \\
y^{\Delta}=-c(t) x(\sigma(t))-a(t) y(t)
\end{array}\right.
$$

and

$$
\left\{\begin{array}{c}
X^{\Delta}=a(t) X(\sigma(t))+b(t) Y(t)+\xi_{1}(t), \\
Y^{\Delta}=-c(t) X(\sigma(t))-a(t) Y(t)+\xi_{2}(t),
\end{array}\right.
$$

where $a(t), \mathrm{b}(t)$ and $c(t)$ are real-valued rd-continuous functions on $\mathrm{T}$ with the coefficient $a(t)$ satisfying the condition

$$
(H): 1-\mu(t) a(t) \neq 0
$$


The system (3.1) represents the master system. The controllers $\xi_{1}(t)$ and $\xi_{2}(t)$ are added into the slave system, given by the system (3.2).

To synchronization the master system (3.1) and the slave system (3.2), the errors systems are designed as follows

$$
\left\{\begin{array}{c}
e_{1}^{\Delta}=a(t) e_{1}(\sigma(t))+b(t) e_{2}(t)+\xi_{1}(t) \\
e_{2}^{\Delta}=-c(t) e_{1}(\sigma(t))-a(t) e_{2}(t)+\xi_{2}(t)
\end{array}\right.
$$

and

$$
\lim _{t \rightarrow \infty}\left|e_{i}\right|=0, i=1,2,
$$

then the synchronization will happen.

Our main result of this paper is as follows

Theorem 3.1 Assume $(\mathrm{H})$ and $\mu>0$ hold, then for given synchronization systems (3.1) and (3.2) will occur by the control law as follows

$$
\left\{\begin{array}{l}
\xi_{1}=-\frac{1}{\mu} e_{1}+\left(\frac{1-a \mu}{2 \mu}-b\right) e_{2} \\
\xi_{2}=\frac{1}{2 \mu} e_{1}+\left(a+\frac{c}{2}-\frac{1}{\mu}\right) e_{2}
\end{array}\right.
$$

where $a, b, c, e_{1}, e_{2}, \mu$ and $\xi_{1}, \xi_{2}$ are the function with respect to $t$.

Proof We take the Lyapunov function as

$$
V=\frac{1}{2}\left(e_{1}^{2}+e_{2}^{2}\right)
$$

satisfies

$$
\left\{\begin{array}{l}
V>0, \quad \text { if }\left(e_{1}, e_{2}\right) \neq(0,0), \\
V=0, \quad \text { if }\left(e_{1}, e_{2}\right)=(0,0) .
\end{array}\right.
$$

Next, applying the (iii) of Lemma 2.2 we have

$$
V^{\Delta}=\frac{1}{2}\left(e_{1}^{2}+e_{2}^{2}\right)^{\Delta}=\frac{1}{2}\left(e_{1} e_{1}^{\Delta}+e_{1}^{\Delta} e_{1}(\sigma(t))+e_{2} e_{2}^{\Delta}+e_{2}^{\Delta} e_{2}(\sigma(t))\right) .
$$

Substituting the formula $f(\sigma(t))=f(t)+\mu(t) f^{\Delta}(t)$ into (3.3) and (3.5), respectively, we have

$$
\left\{\begin{array}{l}
e_{1}^{\Delta}=\frac{a e_{1}+b e_{2}+\xi_{1}}{1-a \mu} \\
e_{2}^{\Delta}=-c \mu \frac{a e_{1}+b e_{2}+\xi_{1}}{1-a \mu}-c e_{1}-a e_{2}+\xi_{2}
\end{array}\right.
$$

and

$$
V^{\Delta}=\frac{1}{2} e_{1}^{\Delta}\left(2 e_{1}+\mu e_{1}^{\Delta}\right)+\frac{1}{2} e_{2}^{\Delta}\left(2 e_{2}+\mu e_{2}^{\Delta}\right)
$$

Substituting

$$
\left\{\begin{array}{l}
\xi_{1}=-\frac{1}{\mu} e_{1}+\left(\frac{1-a \mu}{2 \mu}-b\right) e_{2} \\
\xi_{2}=\frac{1}{2 \mu} e_{1}+\left(a+\frac{c}{2}-\frac{1}{\mu}\right) e_{2}
\end{array}\right.
$$

into (3.6), then it yields 


$$
\left\{\begin{array}{c}
e_{1}^{\Delta}=-\frac{1}{\mu} e_{1}+\frac{1}{2 \mu} e_{2}, \\
e_{2}^{\Delta}=\frac{1}{2 \mu} e_{1}-\frac{1}{\mu} e_{2},
\end{array}\right.
$$

then, substituting (3.8) into (3.7), since $\mu(t)=\sigma(t)-t>0$ we obtain

$$
V^{\Delta}=-\frac{3}{8 \mu}\left(e_{1}^{2}+e_{1}^{2}\right)<0 .
$$

According to the Lyapunov stability theory, the error systems asymptotically tends to zero. Thus, the proof is completely.

Theorem 3.2 Assume $(\mathrm{H})$ and $\mu=0$ hold, then for given synchronization systems (3.1) and (3.2) will occur by the control law as follows

$$
\left\{\begin{array}{l}
\xi_{1}=-(a+1) e_{1}+\frac{c-b}{2} e_{2}, \\
\xi_{2}=\frac{c-b}{2} e_{1}+(a-1) e_{2},
\end{array}\right.
$$

where $a, b, c, e_{1}, e_{2}, \mu$ and $\xi_{1}, \xi_{2}$ are the function with respect to $t$.

Proof When $\mu=0$, the formula turns into $f(\sigma(t))=f(t)$ and system (3.3) is equivalent to

$$
\left\{\begin{array}{c}
e_{1}^{\Delta}=a(t) e_{1}(t)+b(t) e_{2}(t)+\xi_{1}(t), \\
e_{2}^{\Delta}=-c(t) e_{1}(t)-a(t) e_{2}(t)+\xi_{2}(t) .
\end{array}\right.
$$

Similarly we also take the Lyapunov function as

$$
V=\frac{1}{2}\left(e_{1}^{2}+e_{2}^{2}\right)
$$

satisfies

$$
\begin{cases}V>0, & \text { if }\left(e_{1}, e_{2}\right) \neq(0,0), \\ V=0, & \text { if }\left(e_{1}, e_{2}\right) \neq(0,0) .\end{cases}
$$

Since the formula turns into $f(\sigma(t))=f(t)$ for which we have

$$
V^{\Delta}=e_{1} e_{1}^{\Delta}+e_{2} e_{2}^{\Delta} \text {. }
$$

Substituting

into (3.10), then it yields

$$
\left\{\begin{array}{l}
\xi_{1}=-\frac{1}{\mu} e_{1}+\left(\frac{1-a \mu}{2 \mu}-b\right) e_{2} \\
\xi_{2}=\frac{1}{2 \mu} e_{1}+\left(a+\frac{c}{2}-\frac{1}{\mu}\right) e_{2}
\end{array}\right.
$$

$$
\left\{\begin{array}{c}
e_{1}^{\Delta}=-e_{1}+\frac{c+b}{2} e_{2}, \\
e_{2}^{\Delta}=\frac{c+b}{2} e_{1}-e_{2}
\end{array}\right.
$$

then,

$$
V^{\Delta}=-\left(e_{1}^{2}+e_{2}^{2}\right)<0 .
$$

According to the Lyapunov stability theory, the error systems asymptotically tends to zero. Thus, the proof is completely. 


\section{Conclusion}

In this paper, we have studied the synchronization on time scale and given two theorems to illustrate the phenomenon. Furthermore, corresponding examples are presented based on the results. Numerical simulations show their feasibility. From a theoretical perspective, such a synchronization strengthen the security of communication, which could be considered as a good method for designing a more secure communication scheme in practical engineering applications.

\section{References}

[1] Du, H.Y., Zeng, Q.S., Wang, C.H., Ling, M.X. (2010) Function projective synchronization in coupled chaotic systems. Nonlinear Analysis: Real World Applications, 11, 705-712.

[2] An, H., Chen, Y. (2008) The function cascade synchronization method and applications. Communications in Nonlinear Science and Numerical Simulation , 13, 2246-2255.

[3] Chu, Y.D., Chang, Y.X., An, X.L., Yu, J.N., Zhang , J.G. (2011) A new scheme of general hybrid projective complete dislocated synchronization. Commun Nonlinear Sci Numer Simulat, 16 , 1509-1516.

[4] Lu, J.Q., Ho, D. W., Cao, J.D. (2010) A unified synchronization criterion for impulsive dynamical networks, Automatica, 46, 1215-1221.

[5] Ramakrishnan, R., Krishnamurthy, A. (2012) Performance evaluation of asynchronization station with multiple inputs and population constraints. Computers Operations Research, 39, 560570.

[6] Zhang, Q. M., He, X., Jiang, J. (2011) On Lyapunov-type inequalities for nonlinear dynamic systems on time scales. Computers and Mathematics with Applications, 62, 4028-4038.

[7] J.Hoffacker, C., Tisdell, C. (2005) Stability and Instability for Dynamic Equations on Time Scales. Computers and Mathematics with Applications, 49, 1327-1334.

[8] Liu, G., Xiang, X., Peng, Y. (2011) Nonlinear integro-differential equations and optimal control problems on time scales. Computers and Mathematics with Applications, 61, 155-169.

[9] Jiang, L., Zhou, Z. (2005) Lyapunov inequality for linear Hamitonian systems on time scales. Journal of Mathematical Analysis and Applications, 310, 579-593.

[10] Cakmak, D. (2013) On Lyapunov-type inequality for a class of nonlinear systems. Math. Inequal. Appl, 16, 101-108.

[11] Dhar, S., Kong, Q. (2014) Liapunov-type inequalities for third-order half-linear equations and applications to boundary value problems. Nonlinear Analysis: Theory, Methods and Applications, $110,170-181$.

[12] Agarwal, R.P., Özbekler, A. (2015) Lyapunov type inequalities for even order differential equations with mixed nonlinearities. Journal of Inequalities and Applications, 1, 142.

[13] Agarwal, R.P, Özbekler, A. (2015) Lyapunov type inequalities for second order sub-and superhalf-linear differential equations. Dyn. Syst. Appl, 24, 211-220.

[14] Liu, J., Sun, T., Kong, X., et al. (2016) Lyapunov inequalities of linear Hamiltonian systems on time scales. J. Comput. Anal. Appl, 21, 1160-1169.

[15] Agarwal, R.P., Özbekler, A. (2017) Lyapunov type inequalities for mixed nonlinear RiemannLiouville fractional differential equations with a forcing term. Journal of Computational and Applied Mathematics, 314, 69-78.

[16] Sun, T., Zeng, F., Su, G., et al. (2018) Lyapunov inequalities of quasi-Hamiltonian systems on time scales. Journal of Computational Analysis and Applications, 848.

[17] Diethelm, K., Ford, N.J., Freed, A.D. (2003) A predictor-corrector approach for the numerical solution of fractional differential equation. Nonlinear Dynamics, 29, 3-22. 